\title{
A CONTRIBUTION TO RENEWAL THEORY ${ }^{1}$
}

JOHN LAMPERTI

1. Statement of results. Suppose that $X_{i}, i=1,2, \ldots$, are independent, identically distributed positive random variables and that $S_{i}$ are their partial sums, $S_{0}=0$. The random variables

$$
Y_{t}=t-\max \left\{S_{i} \mid S_{i} \leqq t\right\}
$$

represent in renewal theory the elapsed lifetimes of the "item in use" at time $t$. The limiting behavior of $Y_{t}$ as $t \rightarrow \infty$ has been extensively studied; if $\mu=E\left(X_{i}\right)<\infty, Y_{t}$ itself has a limiting distribution [8; 2] while if $\mu=\infty$, the generalized arc-sine laws emerge as the possible limit distributions of $Y_{t} / t[2 ; 6]$. It may be remarked that $Y_{t}$ forms a Markov process with right-continuous path functions.

The purpose of this paper is to analyze similarly the limiting behavior of two other random variables associated with the sequence $\left\{X_{i}\right\}$; they are

$$
M_{t}=\sup _{\tau \leqq t} Y_{\tau} \quad \text { and } \quad T_{x}=\min \left\{\tau \mid Y_{\tau} \geqq x\right\} .
$$

Thinking of $Y_{t}$ as a Markov process, these are the maximum of the path functions up to time $t$ and the first-passage time from 0 to $x$, respectively. In the renewal interpretation $M_{t}$ probably has the greater interest; it represents the maximum lifetime observed up to time $t$, with the final incomplete lifetime $Y_{t}$ an admissible candidate. There is a simple relation between the distributions of $M_{t}$ and $T_{x}$, namely

$$
\operatorname{Pr}\left(T_{x}<t\right)=\operatorname{Pr}\left(M_{t}>x\right),
$$

which means that only one of them need be considered at first to gain information about both. It will be convenient for us to study $T_{x}$ primarily. In both statements and proofs an important role is again played by slowly varying functions. ${ }^{2}$

Let $F(x)=\operatorname{Pr}\left(X_{i} \leqq x\right)$, and assume $F(x)<1$ for every $x<\infty$. The asymptotic behavior of $T_{x}$ is as follows.

Theorem 1. Provided that either $\mu=E\left(X_{i}\right)<\infty$, or $\mu=\infty$ and

Received by the editors October 3, 1960.

1 This work was supported by the National Science Foundation.

${ }^{2}$ A positive function $L(x)$ is slowly varying if $L(c x) / L(x) \rightarrow 1$ as $x \rightarrow \infty$ for every $c>0[4]$. 
$1-F(x)=x^{-1} L(x)$ with $L(x)$ slowly varying, there exists an increasing function $b(x)$ such that

$$
\lim _{x \rightarrow \infty} \operatorname{Pr}\left[T_{x} \leqq b(x) t\right]=1-e^{-t}
$$

In the first case $b(x)$ may be taken to be $\mu[1-F(x)]^{-1}$. However, in order that

$$
\lim _{x \rightarrow \infty} \operatorname{Pr}\left(T_{x} \leqq x t\right)=H(t)
$$

shall exist with $H(t)$ a nondegenerate distribution function, it is necessary and sufficient that $1-F(x)=x^{-\alpha} L(x)$ with $0<\alpha<1$ and $L(x)$ slowly varying. ${ }^{3}$ If this is the case, then $H(t)=H_{\alpha}(t)$ is the distribution with Laplace transform

$$
\hat{H}_{\alpha}(\lambda)=\int_{0}^{\infty} e^{-\lambda t} d H_{\alpha}(t)=\left[1+\lambda \int_{0}^{1} y^{-\alpha} e^{\lambda(1-y)} d y\right]^{-1} .
$$

If $\alpha=0$, (1.5) and (1.6) are still valid.

With the aid of (1.3) we can easily deduce from Theorem 1 some properties of $M_{t}$.

ThEOREM 2. Whenever (1.4) holds,

$$
\lim _{t \rightarrow \infty} \operatorname{Pr}\left[M_{t} \leqq b^{-1}(t / x)\right]=e^{-x}
$$

There is a nondegenerate distribution $G(x)$ such that

$$
\lim _{t \rightarrow \infty} \operatorname{Pr}\left(M_{t} \leqq t x\right)=G(x)
$$

if and only if $1-F(x)=x^{-\alpha} L(x)$ with $0<\alpha<1$ and $L(x)$ slowly varying; if this is the case, then

$$
G(x)=G_{\alpha}(x)=1-H_{\alpha}(1 / x) .
$$

REMARKs. Relation (1.7) can be transformed in certain cases. For instance, if $1-F(x) \sim a x^{-\alpha}$ as $x \rightarrow \infty$ for some $\alpha>1, b(x)$ can be taken to be exactly $\mu a^{-1} x^{\alpha}$ and (1.7) becomes by a change of variable

$$
\lim _{t \rightarrow \infty} \operatorname{Pr}\left[M_{t} \leqq(a t / \mu)^{1 / \alpha} y\right]=\exp \left(-y^{-\alpha}\right), \quad y \geqq 0 .
$$

If instead $1-F(x) \sim a \exp \left[-\left(x^{\alpha}\right)\right], \alpha>0$, we obtain

3 This same condition occurs in the studies of $Y_{t} / t$ referred to above, and in many additional places in the asymptotic theory of Markov and renewal processes; it implies that $X_{i}$ belongs to the domain of attraction of a stable law. 


$$
\lim _{t \rightarrow \infty} \operatorname{Pr}\left[M_{t}^{\alpha} \leqq \log (a t / \mu)+y\right]=\exp \left(-e^{-y}\right) .
$$

These distributions are also limit distributions for the maximum term in a sequence of $n$ independent, identically distributed random variables [3]. This shows that the cases with $\mu<\infty$ in Theorems 1 and 2 are rather obvious. The (random) number $N_{t}$ of renewals before time $t$ is approximately $t / \mu$ in this situation; if this number were known exactly and if it were not necessary to impose the condition $S_{N_{t}} \leqq t,(1.4)$ and its consequences would be immediate. While (1.4) could no doubt be proved rigorously along these lines, we shall use another approach which handles the cases $\mu<\infty$ and $\mu=\infty$ in a unified way.

Some properties of the laws $H_{\alpha}(t)$ and a method of inverting (1.6) are discussed in $\$ 3$. A few moments, of course, are readily obtained from (1.6) and it is not difficult to show also that when $1-F(x)$ $=x^{-\alpha} L(x), \alpha<1$,

$$
\begin{gathered}
\lim _{x \rightarrow \infty} E\left(T_{x} / x\right)=\int_{0}^{\infty} t d H_{\alpha}(t)=\frac{1}{1-\alpha}, \\
\lim _{x \rightarrow \infty} E\left(T_{x}^{2} / x^{2}\right)=\int_{0}^{\infty} t^{2} d H_{\alpha}(t)=\frac{2}{(1-\alpha)^{2}(2-\alpha)} .
\end{gathered}
$$

Similar results for higher moments could also be obtained.

These problems and, in a general way, the method of attacking them were suggested by work of Karlin and McGregor [5], who studied the maximum functional for birth-and-death processes. The author is also deeply indebted to G. Latta, to whose suggestions the results of the last section are due.

2. Proof of the theorems. We start with the generating function of $T_{x}$, and use the customary "renewal" argument. That is, if $X_{1}>x$, then $e^{-\lambda T_{x}}=e^{-\lambda x}$, while if $X_{1} \leqq x$ the process starts over at time $X_{1}$, $T_{x}$ having received in advance a contribution of $X_{1}$. This gives

$$
E\left(e^{-\lambda T_{x}}\right)=e^{-\lambda x}[1-F(x)]+\int_{0}^{x} E\left(e^{-\lambda\left(T_{x}+y\right)}\right) d F(y) .
$$

Solving we obtain

$$
E\left(e^{-\lambda T x}\right)=\frac{e^{-\lambda x}[1-F(x)]}{1-\int_{0}^{x} e^{-\lambda y} d F(y)} .
$$


Introducing an unspecified normalizing function $b(x)$ and integrating by parts in the denominator yields

$$
\begin{aligned}
& E\left(e^{-\lambda T_{x} / b(x)}\right) \\
& \quad=\left[1+\lambda \frac{e^{\lambda x / b(x)}}{b(x)[1-F(x)]} \int_{0}^{x}[1-F(y)] e^{-\lambda y / b(x)} d y\right]^{-1} .
\end{aligned}
$$

To obtain (1.4), we put

$$
b(x)=\frac{\int_{0}^{x}[1-F(y)] d y}{1-F(x)},
$$

and assume for the moment that

$$
\lim _{x \rightarrow \infty} \frac{x}{b(x)}=0 .
$$

Then the exponential terms in the right-hand side of (2.2) tend to one, the second one uniformly over the range of integration, and

$$
\lim _{x \rightarrow \infty} E\left(e^{-\lambda T_{x} / b(x)}\right)=(1+\lambda)^{-1} .
$$

In view of the continuity theorem for Laplace-Stieltjes transforms (2.5) implies (1.4), which therefore holds whenever $b(x)$ as defined in (2.3) satisfies (2.4). This must be so if $\mu<\infty$, for then $1-F(x)$ $=o(1 / x)$ and $\int_{0}^{\infty}[1-F(y)] d y=\mu$. In case $1-F(x)=x^{-1} L(x)$ and $\mu=\infty$, we argue as follows:

$$
\frac{x}{b(x)}=\frac{L(x)}{\int_{0}^{x} y^{-1} L(y) d y} \leqq \frac{L(x)}{\int_{\epsilon x}^{x} y^{-1} L(y) d y}, \quad 1>\epsilon>0,
$$

since the integrand is non-negative. The last expression is the same as $L(x) / \int_{\epsilon}^{1} t^{-1} L(x t) d t$, and it is a property of slowly varying functions that the convergence of $L(c x) / L(x)$ to 1 is uniform if $x$ is bounded from 0 and from $\infty[4]$. We thus obtain

$$
\limsup _{x \rightarrow \infty} \frac{x}{b(x)} \leqq \frac{1}{\log 1 / \epsilon},
$$

and since $\epsilon$ is arbitrarily small this gives (2.4). The first part of Theorem 1 is established.

To study the validity of (1.5) we put $b(x)=x$ in (2.2), and find, again by the continuity theorem, that (1.5) holds if and only if 


$$
\lim _{x \rightarrow \infty} \frac{\int_{0}^{x}[1-F(y)] e^{-\lambda y / x} d y}{x[1-F(x)]} \quad \text { exists, } \quad \lambda>0,
$$

is not $\not \equiv 0$, and is $o\left(\lambda^{-1}\right)$ as $\lambda \rightarrow 0$. Provided $1-F(x)=x^{-\alpha} L(x), \alpha<1$, (2.6) becomes

$$
\lim _{x \rightarrow \infty} \frac{\int_{0}^{1} t^{-\alpha} e^{-\lambda t} L(x t) d t}{L(x)},
$$

and by a theorem in [1], this limit does exist and equals $\int_{0}^{1} t^{-\alpha} e^{-\lambda t} d t$. This is bounded as $\lambda \rightarrow 0$ so $E\left(e^{-\lambda T_{x} / x}\right)$ converges to the transform of a distribution, proving sufficiency in the second part of Theorem 1.

For the converse, begin with (2.6) in the form

$$
\lim _{x \rightarrow \infty} \int_{0}^{1} \frac{[1-F(x t)]}{1-F(x)} e^{-\lambda t} d t \text { exists, } \quad \lambda>0 .
$$

In (2.7) we have the Laplace-Stieltjes transforms of the increasing functions

$$
\int_{0}^{y} \frac{[1-F(x t)]}{1-F(x)} d t=K_{x}(y), \quad y \leqq 1 .
$$

Since $e^{-\lambda t}$ is bounded below on $[0,1]$ for fixed $\lambda$, the existence of the limit in (2.7) implies first that $K_{x}(1)$ is bounded as $x \rightarrow \infty$ and so also that $K_{x}(y)$ converges. It follows that we are justified in putting $\lambda=0$ in (2.6) and obtain a positive limit. If now $f(x)=\int_{0}^{x}[1-F(y)] d y$, we have

$$
\lim _{x \rightarrow \infty} \frac{x f^{\prime}(x)}{f(x)}=c \text { exists. }
$$

By Theorem 2 of $[7]^{4}$ it follows that $f(x)=x^{c} L(x)$, and these facts together give

$$
f^{\prime}(x)=1-F(x)=x^{-\alpha} L_{1}(x)
$$

with $\alpha=1-c$ and $L_{1}(x)$ another slowly varying function. If $\alpha \geqq 1$, we have seen in the first part of the proof that $b(x)$ is larger order than $x$; $\alpha$ cannot be negative and $\alpha=0$ leads to a degenerate distribution. Hence $0<\alpha<1$, and the proof of necessity is complete.

The convergence of moments stated in (1.12) and (1.13) does not, of

4 This theorem is stated in terms of $x \rightarrow 0$; the proof applies without change to the case $x \rightarrow \infty$. 
course, follow from what has been proved so far, but can be demonstrated by very similar methods. Differentiating (2.2) with $b(x)=x$ yields

$$
E\left(T_{x} / x\right)=\frac{\int_{0}^{x}[1-F(y)] d y}{x[1-F(x)]} .
$$

In case $1-F(x)=x^{-\alpha} L(x)$, (2.8) implies (1.12) by, for instance, another use of Theorem 2 of [7]. With slightly more labor, the second and higher asymptotic moments can be evaluated in the same way.

A few remarks will suffice for Theorem 2. First, (1.3) follows from the identity of the events $T_{x}<t$ and $M_{t}>x$; this in turn depends on right-continuity of the (random) function $Y_{t}$. (The strict inequalities are essential if (1.3) is to be valid in all cases.) The use of (1.3) to convert (1.4) into (1.7) and (1.5) into (1.9) is both simple and familiar; the resulting limit statements hold at all continuity points of the original limit distribution. (In fact, as we shall see, the distributions $H_{\alpha}(t)$ are continuous except in the case $\alpha=0$.) Finally, we comment that (1.10) could be slightly generalized by including a slowly varying function in the asymptotic expression for $1-F(x)$.

3. The distribution $H_{\alpha}(t)$. The functions $H_{\alpha}(t)$ are determined at continuity points by (1.6) and the requirement that they be nondecreasing. We rewrite (1.6) in the form

$$
\frac{e^{-\lambda}}{\lambda} \hat{H}_{\alpha}(\lambda)+\int_{0}^{1} e^{-\lambda x} x^{-\alpha} d x \hat{H}_{\alpha}(\lambda)=\frac{e^{-\lambda}}{\lambda},
$$

and observe that since

$$
\frac{e^{-\lambda}}{\lambda}=\int_{0}^{\infty} e^{-\lambda x} d f(x), \quad f(x)= \begin{cases}0, & x \leqq 1 \\ x-1, & x>1\end{cases}
$$

both terms on the left in (3.1) are products of Laplace-Stieltjes transforms. Inverting therefore leads to Stieltjes convolutions:

$$
\int_{0}^{t} H_{\alpha}(t-x) d f(x)+\int_{0}^{t} H_{\alpha}(t-x) d g_{\alpha}(x)=f(x),
$$

where $g_{\alpha}(x)=\int_{0}^{\min 1, x} y^{-\alpha} d y$. If $t \leqq 1$ (3.3) becomes

$$
\int_{0}^{t} H_{\alpha}(y)(t-y)^{-\alpha} d y=0
$$

while for $t>1$, 


$$
\int_{0}^{t-1} H_{\alpha}(y) d y+\int_{t-1}^{t} H_{\alpha}(y)(t-y)^{-\alpha} d y=t-1=f(t) .
$$

Introducing the fractional integration operator

$$
I^{\alpha} F(t)=\frac{1}{\Gamma(\alpha)} \int_{0}^{t} F(x)(t-x)^{\alpha-1} d x,
$$

(3.4) becomes $\Gamma(1-\alpha) I^{1-\alpha} H_{\alpha}(t)=0$ for $t \leqq 1$ from which we obtain (apply $I^{\alpha}$ to both sides) that $H_{\alpha}(t)=0, t \leqq 1$. (This is obvious from the probabilistic context.) On the other hand, (3.5) can be written as (3.6) $\Gamma(1-\alpha) I^{1-\alpha} H_{\alpha}(t)=f(t)+\int_{0}^{t-1} H_{\alpha}(y)\left\{(t-y)^{-\alpha}-1\right\} d y, t>1$.

From this equation $H_{\alpha}(t)$ can be computed step-by-step, for the righthand side depends on knowledge of $H_{\alpha}(y)$ only for $y \leqq t-1$. For instance, since $H_{\alpha}(y)=0$ for $y \leqq 1$ the last term in (3.6) vanishes if $1 \leqq t \leqq 2$. Therefore, in that interval,

$$
I H_{\alpha}(t)=\frac{1}{\Gamma(1-\alpha)} I^{\alpha} f(t)=\frac{\int_{1}^{t}(t-x)^{\alpha-1}(x-1) d x}{\Gamma(\alpha) \Gamma(1-\alpha)}
$$

from which we obtain

$$
H_{\alpha}(t)=\frac{\sin \pi \alpha}{\pi \alpha}(t-1)^{\alpha} \quad \text { for } 1 \leqq t \leqq 2 .
$$

The general formula is, for $t \geqq 2$,

$$
H_{\alpha}(t)=\frac{\sin \pi \alpha}{\pi \alpha}(t-1)^{\alpha}+\frac{1}{\Gamma(1-\alpha)} \frac{d}{d t}\left\{I^{\alpha} g(t)\right\},
$$

where $g(t)$ is the last term in (3.6), zero if $t \leqq 2$.

It seems difficult to carry out an explicit calculation of $H_{\alpha}(t)$ for all $t$. Nevertheless the iterative scheme yields some more information: $H_{\alpha}(t)$ is absolutely continuous for all $\alpha \in(0,1)$, and its density $h_{\alpha}(t)$ is continuous for $t \neq 1$. The proof is not difficult; the statements follow from (3.7) for $t<2$. If $H_{\alpha}$ is continuous up to $t-1 / 2$, say, it is readily verified that $g$ must be twice continuously differentiable up to $t$. From (3.8) then follows that $H_{\alpha}^{\prime}$ exists and is continuous up to $t$ except for the infinity at $t=1$. Continuing in steps of $1 / 2$ (or any amount between 0 and 1) the existence and continuity of $H_{\alpha}^{\prime}(t)$ is proved for all $t>1$. Absolute continuity follows since (3.7) shows that there is no discontinuity of $H_{\alpha}$ at $t=1$. 


\section{REFERENCES}

1. S. Aljančić, R. Bojanić, and M. Tomić, Sur la valeur asymptotique d'une classe des intégrales définies, Acad. Serbe Sci. Publ. Inst. Math. vol. 7 (1954) pp. 81-94.

2. E. B. Dynkin, Limit theorems for sums of independent random quantities, Izv. Akad. Nauk SSSR vol. 19 (1955) pp. 247-266 (Russian).

3. E. J. Gumbel, Statistics of extremes, New York, Columbia University Press, 1958.

4. J. Karamata, Sur une mode de croissance regulière des fonctions, Mathematica (Cluj) vol. 4 (1930) pp. 38-53.

5. S. Karlin and J. McGregor, Occupation time laws for birth and death processes, Technical Report No. 24, Contract Nonr-225(28), Stanford University, July 29, 1960.

6. J. Lamperti, Some limit theorems for stochastic processes, J. Math. Mech. vol. 7 (1958) pp. 433-450.

7. - An occupation time theorem for a class of stochastic processes, Trans. Amer. Math. Soc. vol. 88 (1958) pp. 380-387.

8. W. L. Smith, Asymptotic renewal theorems, Proc. Roy. Soc. Edinburgh. Sect. A vol. 64 (1954) pp. 9-48.

STANFORD UNIVERSITY

\section{ON THE INTERTWINING NUMBER THEOREM}

\section{ADAM KLEPPNER}

Let $G_{1}$ and $G_{2}$ be open subgroups of the separable locally compact group \& and let $L$ and $M$ be unitary representations of $G_{1}$ and $G_{2}$ respectively. For each $x$ and $y$ in $G$ the indices of the subgroup $H_{x, y}$ $=x^{-1} G_{1} x \cap y^{-1} G_{2} y$ in $x^{-1} G_{1} x$ and in $y^{-1} G_{2} y$ depend only on the $G_{1}: G_{2}$ double coset to which $x y^{-1}$ belongs. Let $\mathfrak{D}_{f}$ denote the set of all $G_{1}: G_{2}$ double cosets for which both these indices are finite and let $\mathfrak{D}$ denote the set of all $G_{1}: G_{2}$ double cosets. Let $\Im(L, M ; x, y)$ denote the intertwining number of the representations $s \rightarrow L_{x s x^{1}}=L_{s}^{x}$ and $s \rightarrow M_{y s y}{ }^{-1}$ $=M_{s}^{v}$ of $H_{x, y}$. The Intertwining Number Theorem of Mackey [1, Theorem $3^{\prime}$ ] states the following:

(1) $\Im(L, M ; x, y)$ depends only on the $G_{1}: G_{2}$ double coset $D$ to which $x y^{-1}$ belongs and may be denoted by $\mathfrak{S}(L, M ; D)$;

$$
\sum_{D \in D_{f}} \Im(L, M ; D) \leqq \Im\left(U^{L}, U^{M}\right) \leqq \sum_{D \in \mathscr{D}} \Im(L, M ; D) ;
$$

(3) if $L$ and $M$ are 1-dimensional then $\Im\left(U^{L}, U^{M}\right)=\sum_{D \in \mathfrak{D}_{f}} \Im(L, M ; D)$.

Received by the editors May 23, 1960. 